\title{
Ground States, Excited States, and Metal-Ligand Bonding in Rare Earth Hexachloro Complexes: A DFT-Based Ligand Field Study
}

\author{
M. Atanasov, ${ }^{\star, t, \neq ~ C . ~ D a u l, ~}{ }^{\star, \dagger}$ H. U. Gudel, $\$$ T. A. Wesolowski," and M. Zbiri ${ }^{\dagger}$ \\ Département de Chimie, Université de Fribourg Suisse, Ch. du Musée 9, \\ CH-1700 Fribourg, Switzerland, Institute of General and Inorganic Chemistry, \\ Bulgarian Academy of Sciences, Acad.G.Bontchev Str. Bl.11, 1113 Sofia, Bulgaria, Department of \\ Chemistry and Biochemistry, University of Bern, Freiestrasse 3, CH-3012 Bern, Switzerland, and \\ Département de Chimie Physique, Université de Genève, Sciences II, 30, Quai Ernest-Ansermet, \\ CH-1211 Genève 4, Switzerland
}

Received September 8, 2004

Metal (4f)-ligand $(\mathrm{Cl} 3 p)$ bonding in $\mathrm{LnCl}_{6}{ }^{3-}(\mathrm{Ln}=\mathrm{Ce}$ to $\mathrm{Yb})$ complexes has been studied on the basis of $4 \mathrm{f} \rightarrow 4 \mathrm{f}$ and $\mathrm{Cl}, 3 \mathrm{p} \rightarrow 4 \mathrm{f}$ charge-transfer spectra and on the analysis of these spectra within the valence bond configuration interaction model to show that mixing of $\mathrm{Cl} 3 p$ into the $\mathrm{Ln} 4 \mathrm{f}$ ligand field orbitals does not exceed $1 \%$. Contrary to this, Kohn-Sham formalism of density functional theory using currently available approximations to the exchangecorrelation functional tends to strongly overestimate $4 \mathrm{f}-3 p$ covalency, yielding, for $\mathrm{YbCl}_{6}{ }^{3-}$, a much larger mixing of $\mathrm{Cl} 3 p \rightarrow 4 f$ charge transfer into the $f^{13}$ ionic ground-state wave function. Thus, ligand field density functional theory, which was recently developed and applied with success to complexes of $3 \mathrm{~d}$ metals in our group, yields anomalously large ligand field splittings for $L n$, the discrepancy with experiment increasing from left to the right of the $\mathrm{Ln} 4 \mathrm{f}$ series. It is shown that eliminating artificial ligand-to-metal charge transfer in Kohn-Sham calculations by a procedure described in this work leads to energies of $4 \mathrm{f}-4 \mathrm{f}$ transitions in good agreement with experiment. We recall an earlier concept of Ballhausen and Dahl which describes ligand field in terms of a pseudopotential and give a thorough analysis of the contributions to the ligand field from electrostatics (crystal field) and exchange (Pauli) repulsion. The close relation of the present results with those obtained using the first-principles based and electron density dependent effective embedding potential is pointed out along with implications for applications to other systems.

\section{Introduction}

In this work we study metal-ligand bonding in octahedral rare earth lanthanides $(\mathrm{Ln})$ hexachloro complexes, $\mathrm{LnCl}_{6}{ }^{3-}(\mathrm{Ln}$ $=\mathrm{Ce}^{3+}$ to $\left.\mathrm{Yb}^{3+}\right)$. We aim at developing a computational scheme within the ligand field model, based on Kohn-Sham DFT orbitals ${ }^{1}$ capable of predicting with reasonable accuracy energies of $\mathrm{f} \rightarrow \mathrm{f}$ transitions. We will use the label KSDFT to denote the Kohn-Sham DFT with an exchange-correlation functional chosen in the form of the generalized gradient approximation.

The optical and magnetic properties of Ln have been intensively studied because of their unique applications for

\footnotetext{
* Authors to whom correspondence should be addressed. E-mail: mihail.atanasov@unifr.ch (M.A.), claude.daul@unifr.ch (C.D.).

$\dagger$ Université de Fribourg Suisse.

$\div$ Bulgarian Academy of Sciences.

$\S$ University of Bern.

"Université de Genève.

(1) Kohn, W.; Sham, L. J. Phys. Rev. 1965, 140, A1133.
}

2954 Inorganic Chemistry, Vol. 44, No. 8, 2005 a rational design of molecular devices with predetermined optical (fluoroimmunoassays, near-infrared emitters, and analytical sensors) ${ }^{2}$ and magnetic (smart and responsive medical magnetic resonance imaging contrast agents) ${ }^{3}$ functions.

Correlations between molecular structures, crystal field parameters and the electronic properties allow an ultra-finetuning of the electronic levels in Ln complexes. ${ }^{4}$ Electronic multiplets within the highly localized $\mathrm{f}^{\mathrm{n}}$ configuration of the Ln have been successfully described using various modifications of crystal field theory (CFT),${ }^{5-7}$ or within the angular overlap model (AOM $)^{8,9}$ both models introducing adjustable parameters which can be determined by fitting electronic spectra with high resolution. However, with increasing complexity of Ln compounds when including more than one coordinating ligand and/or coordination geometries of low symmetry, the number of parameters in both models start to exceed the number of observables. For such systems, the necessity of using first-principles, parameter-free computa10.1021/ic040105t CCC: $\$ 30.25$ C 2005 American Chemical Society Published on Web 03/23/2005 
tions become increasingly pronounced in recent time. Split levels of lanthanide monoxides and monofluorides were examined in terms of energy-adjusted first-principles (quasirelativistic) pseudopotentials, ${ }^{10}$ and an improved method has been successfully applied to $\mathrm{LnX}(\mathrm{Ln}=\mathrm{Ce}, \mathrm{X}=\mathrm{O}, \mathrm{F})$ diatomics. ${ }^{10 \mathrm{c}}$ However, a pseudopotential-based LF treatment did not give reliable parameters, due to numerical inaccuracies. ${ }^{10 \mathrm{c}}$ A MCSCF-CI study on $\mathrm{LaO}$ has been reported and the results were compared with LF caculations. ${ }^{11}$ HartreeFock results, which provide the starting point for MCSCF calculations, are not accurate enough in these electronically highly correlated systems and computational efforts are too large to be applied for larger molecules.

One way to circumvent the difficulty of calculating the ligand fields (LF) using ab initio methods is to introduce semiempirical models, to allow calculations of both geometries and $\mathrm{f} \rightarrow \mathrm{f}$ excitation energies. Models of this type can be applied to interpret spectra in solutions where little or no structural information about the active coordination site is known. Such computational schemes have already been developed as given, cf. the Sparkle model for the calculation

(2) (a) Sabbatini, N.; Guardigli, M.; Lehn, J.-M. Coord. Chem. Rev. 1993, 123, 201. (b) Sabbatini, N.; Guardigli, M.; Manet, I. In Handbook on the Physics and Chemistry of Rare Earths; Gschneidner, K. A., Jr., Eyring, L. Eds.; Elsevier: Amsterdam, 1996; Vol. 23, pp 69-119. (c) Werts, M. H. V.; Woudenberg, R. H.; Emmerink, P. G.; van Gassel, R.; Hofstraat, J. W.; Verhoeven, J. W. Angew. Chem., Int. Ed. 2000 39, 4542. (d) Mathis, G. In Rare Earths; Saez Puche, R., Caro, P., Eds.; Editorial Complutense S.A.: Madrid, 1998; pp 285-298. (e) Parker, D. Coord. Chem. Rev. 2000, 205, 109.

(3) (a) Caravan, P.; Ellison, J. J.; McMurry, T. J.; Lauffer, R. B. Chem. Rev. 1999, 99, 2293. (b) The Chemistry of Contrast Agents in Medical Magnetic Resonance Imaging, Meerbach, A. E., Toth, E., Eds.; John Wiley: London, 2001. (c) Lowe, M. P. Aust. J. Chem. 2002, 55, 551. (d) Aime, S.; Castelli, D. D.; Fedeli, F.; Terreno, E. J. Am. Chem. Soc. 2002, 124, 9364. (e) Parker, D.; Dickins, R. S.; Puschmann, H.; Crossland, C.; Howard, J. A. K. Chem. Rev. 2002, $102,1977$.

(4) (a) Carnall, W. T. In Handbook of the Physics and Chemistry of Rare Earths; Gschneidner, K. A. Jr., Eyring, L. Eds.; North-Holland Publishing Company: Amsterdam, 1979; pp 171-208 (b) GörllerWalrand, C.; Binnemans, K. In Handbook of the Physics and Chemistry of Rare Earths; Gschneidner, K. A., Jr., Eyring, L. Eds.; North-Holland Publishing Company: Amsterdam, 1996; Vol. 23, pp 121-183. (c) Porcher, P. In Rare Earths; Saez Puche, R., Caro, P., Eds.; Editorial Complutense S.A.; Madrid, 1998; pp 43-66. (d) Görller-Walrand, C.; Binnemans, K. In Handbook of the Physics and Chemistry of Rare Earths; Gschneidner, K. A., Jr., Eyring, L., Eds.; North-Holland Publishing Company: Amsterdam, 1998; Vol. 25, pp 101-264. (e) Mironov, V. S.; Galyametdinov, Y. G.; Ceulemans, A.; GörllerWalrand, C.; Binnemans, K. J. J. Chem. Phys. 2002, 116, 4673.

(5) (a)Judd, B. R. Phys. Rev. 1962, 127, 750. (b) Ofelt, G. S. J. Chem. Phys. 1962, 37, 511.

(6) Lea, K. R.; Leask, M. J. M.; Wolf, W. P. J. Phys. Chem. Solids 1962, 23,1381 .

(7) (a) Newman, D. J. Adv. Phys. 1971, 20, 197. (b) Field, R. W. Ber. Bunsen-Ges. Phys. Chem. 1982, 86, 771. (c) Mulak, J.; Gajek, Z. The Effective Crystal Field Potential; Elsevier: Amsterdam, 2000.

(8) Jørgensen, C. K.; Pappalardo, R.; Schmidtke, H.-H. J. Chem. Phys. 1963, 39, 1422 .

(9) (a) Urland, W. Chem. Phys. 1976, 14, 393. (b) Urland, W. Chem. Phys. Lett. 1981, 77, 58. (c) Urland, W. Chem. Phys. Lett. 1981, 83, 116.

(10) (a) Dolg, M.; Stoll, H.; Savin, A.; Preuss, H. Theor. Chim. Acta 1989, 75, 173. (b) Dolg, M.; Stoll, H.; Preuss, H. J. Chem. Phys. 1989, 90, 1730. (c) Dolg, M.; Stoll, H.; Preuss, H. Theor. Chim. Acta 1993, 85 , 441. From the three CF parameters $-B_{20}, B_{40}$, and $B_{60}$ of $\mathrm{CeO}$ and $\mathrm{CeF}$-only the value of $B_{20}$ has been determined from a molecular pseudopotential calculation. $B_{40}$ and $B_{60}$ have been fixed approximating $B_{40} / B_{20}$ and $B_{60} / B_{20}$ ratios by all-electron quasirelativistic calculations for $\mathrm{Ce}^{2+}$ and $\mathrm{Ce}^{1+}$, respectively.

(11) Schamps, J.; Bencheikh, M.; Barthelat, J.-C.; Field, R. W. J. Chem. Phys. 1995, 103, 8004. of Ln complexes (SMLC) ${ }^{12}$ (and its improvement, SMLC $\mathrm{II}^{13}$ ) and the simple overlap model,,${ }^{14,15}$ both applied with success to obtain geometries and energies of $\mathrm{f}-\mathrm{f}$ transitions in complexes of $\mathrm{Eu}^{3+}, \mathrm{Nd}^{3+}, \mathrm{Gd}^{3+}$, and $\mathrm{Tb}^{3+}$. A semiempirical intermediate neglect of differential overlap method with adaption to spectroscopy (INDO/S) has also been proposed. ${ }^{16}$

KSDFT became increasingly popular in recent time. As manifested by the groups of Baerends, Ziegler, ${ }^{17,18}$ and Daul, ${ }^{19}$ it is able to predict both ground and excited states of transition metal (TM) complexes. Later, it was shown, using $\mathrm{LnF}$ as models $\left(\mathrm{Ln}=\mathrm{Ce},{ }^{20 \mathrm{a}} \mathrm{Pr}-\mathrm{Yb}^{20 b}\right)$, that energy levels of lanthanides can be calculated reasonably well by adopting pseudopotentials for the $4 \mathrm{f}$ electrons obtained using KSDFT. ${ }^{20 \mathrm{a}}$ Accurate density functionals for a pseudopotential are not known yet; therefore, three experimentally adjustable parameters defining the pseudopotential for each metalligand pair have been introduced in ref 20a. The parameters of this pseudopotential parametrization scheme have been regarded as more transferable than the usual ones (CFT or AOM).

Recently, a new approach has been developed in our group. ${ }^{21}$ It is based on a multideterminant description of the multiplet structure originating from the well-defined $\mathrm{d}^{n}$ configuration of a TM in the environment of coordinating ligands by combining the configuration interaction $(\mathrm{CI})$ and the KSDFT approaches. In doing so, both dynamical (via the DFT exchange-correlation potential) and nondynamical (via CI) correlation is introduced, the latter accounting for the rather localized character of the d- or f-electron wave function. The key feature of this approach is the explicit treatment of near degeneracy effects (long-range correlation) using ad hoc CI within the active space of Kohn-Sham (KS) orbitals with dominant $\mathrm{d}$ or $\mathrm{f}$ character. The calculation of the CI matrixes is based on the symmetry decomposition and/or the ligand field analysis of the energies calculated

(12) de Andrade, A. V. M.; da Costa, N. B., Jr.; Simas, A. M.; de Sá, G. F. Chem. Phys. Lett. 1994, 227, 349.

(13) Rocha, G. B.; Freire, R. O.; da Costa, N. B., Jr.; de Sá, G. F.; Simas, A. M. Inorg. Chem. 2004, 43, 2346.

(14) (a) Malta, O. Chem. Phys. Lett. 1982, 87, 27. (b) Albuquerque, R. Q.; Rocha, G. B.; Malta, O. L.; Porcher, P. Chem. Phys. Lett. 2000, 331, 519.

(15) Porcher, P.; Dos Santos, M. C.; Malta, O. Phys. Chem. Chem. Phys. 1999, 1, 397.

(16) (a) Li. L.; Ren, G.; Xu, G.; Wang, X. Int. J. Quantum. Chem. 1983 23, 1305. (b) Culberson, J. C.; Knappe, P.; Rösch, N.; Zerner, M. C. Theor. Chim. Acta 1987, 71, 21. (c) Kotzian, M.; Rösch, N. J. Mol. Spectrosc. 1991, 147, 346. (d) Kotzian, M.; Rösch, N.; Zerner, M. C. Theor. Chim. Acta 1992 81, 201. (e) Kotzian, M.; Rösch, N. Eur. J. Solid State Inorg. Chem. 1991, 28, 127.

(17) te Velde, G.; Bickelhaupt, F. M.; Baerends, E. J.; Fonesca Guerra, C.; van Gisbergen, S. J. A.; Snijders, J. G.; Ziegler, T. J. Comput. Chem. 2001, 22, 931.

(18) Ziegler, T. Chem. Rev. 1991, 91, 651.

(19) Daul, C. Int. J. Quantum Chem. 1994, 52, 867

(20) (a) Dai, D.; Li L.; Ren, J.; Whangbo, M.-H. J. Chem. Phys. 1998, 108, 3479; two parameters, $a$ and $b$, have been introduced to account for the interaction of the $4 \mathrm{f}$ with the ligand core electrons. A third one, $k$, does describe the influence of all valence electrons on the pseudopotential (Pauli repulsion) acting on the reference $4 \mathrm{f}$ electron. (b) Ren, J.; Whangbo, M.-H.; Dai, D.; Li, L. J. Chem. Phys. 1998, 108,8479 .

(21) (a) Atanasov, M.; Daul, C. A.; Rauzy, C. Chem. Phys. Lett. 2003, 367, 737. (b) Atanasov, M.; Daul, C. A.; Rauzy, C. Struct. Bonding 2004, 106, 97. 
according to KSDFT of the single determinants (microstates) constructed from frozen Kohn-Sham orbitals. The latter have been calculated for an average over the $\mathrm{d}^{n}$ or $\mathrm{f}^{n}$ configuration, possibly with fractional occupations of the TM $\mathrm{d}$ or $\mathrm{f}$ orbitals. This procedure yields multiplet energies of TM complexes with an accuracy of $\pm 1000 \mathrm{~cm}^{-1}$. Currently, the procedure has been extended to spin-orbit coupling 22 and allows also to treat zero field splitting (ZFS). ${ }^{23}$ The first applications of this ligand field KSDFT (LFDFT) approach to Ln complexes run into problems, however. Thus, for example, a KSDFT calculation on octahedral $\mathrm{YbCl}_{6}{ }^{3-}$ using Slater type orbitals of triple- $\zeta$ quality for $4 \mathrm{f}$ and the PW91(ADF) functional yields the correct order of orbital energies $\mathrm{a}_{2 \mathrm{u}}<\mathrm{t}_{2 \mathrm{u}}<\mathrm{t}_{1 \mathrm{u}}$ (see section III.2) but a far too large ligand field splitting compared to experiment. ${ }^{24}$ Likewise, the zerofield splitting of the ${ }^{8} \mathrm{~S}_{7 / 2}$ ground state of $\mathrm{Gd}\left(\mathrm{H}_{2} \mathrm{O}\right)_{8}{ }^{3+}$ has been calculated 1 order of magnitude larger than experiment. ${ }^{25}$ It was claimed that fully relativistic calculations (using a four-component DFT method) can give improved excitation energies for $\mathrm{YbH}, \mathrm{YbF}$, and $\mathrm{YbO} .{ }^{26}$ However, artificial spurious splittings of the $\mathrm{Yb} 4 \mathrm{f}^{13}$ orbitals have been encountered and therefore empirical corrections introduced. Does KSDFT work always so badly for Ln complexes and if this is the case how to rectify discrepancies between theory and experiment and to improve the model?

In this paper we study systematically the whole Ln series. We analyze the complete set of spectral data, $\mathrm{f}-\mathrm{f}$ and ligandto-metal charge-transfer transitions (LMCT), using the socalled valence-bond configuration interaction (VBCI) model $^{27}$ and by KSDFT. The effect of Ln-ligand bonding and its influence on the ground and excited states is studied using $\mathrm{LnCl}_{6}{ }^{3-}$ complexes as models. In section II we briefly describe our KSDFT based LF models (section II.1), the oneelectron levels of Ln in octahedral field (section II.2) and give more details about the KSDFT computations in section II.3. In section III.1 we perform an analysis within the VBCI model of $4 \mathrm{f} \rightarrow 4 \mathrm{f}$ and ligand-to-metal charge-transfer spectra allowing to get for the first time reliable estimates of the extent of $4 \mathrm{f}-3 \mathrm{p} \mathrm{Ln}-\mathrm{Cl}$ mixing. In section III. 2 we compare these results with calculations using KSDFT and explore in more detail the failure of the existing DFT functionals to correctly describe ligand-to-metal covalency. By eliminating artificial charge transfer contributions in sections II.1.2. and III.3 we present a computational scheme which allows to nicely reproduce ligand field splitting in $\mathrm{LnCl}_{6}{ }^{3-}$ complexes. An analysis of the results will be given in section III.4 and the close relation of our approach with the pseudopotential concept $^{28}$ for the LF will be stressed. A discussion of the

(22) Atanasov, M.; Rauzy, C.; Baetig, P.; Daul, C. A. Int. J. Quantum Chem. 2005, 102, 119 .

(23) Atanasov, M.; Daul, C. A. C. R. Chim., submitted for publication.

(24) Atanasov, M.; Daul, C. A.; Güdel, H.-U. Computational Chemistry: Reviews of Current Trends; Leszczynski, J., Ed.; World Scientific Publ. Company: Singapore, 2004; Vol. 9.

(25) Borel, A.; Helm, L.; Daul, C. A. Chem. Phys. Lett. 2004, 383, 584

(26) Liu, W.; Dolg, M.; Li, L. J. Chem. Phys. 1998, 108, 2886

(27) (a) Tuczek, F.; Solomon, E. I. Coord. Chem. Rev. 2001, 219-221, 1075. (b) Tuczek, F.; Solomon, E. I. The VBCI Model: CT Transitions of Bridged Dimers; Lever, A. B. P., Ed.; Comprehensive Coordination Chemistry II; Elsevier: New York, 2003; Vol. 2. (c) Zaanen, J.; Sawatzky, G. A. Can. J. Phys. 1987, 65, 1262. contribution to the pseudopotential from electrostatic (Coulombic) metal-ligand interactions and repulsive (Pauli) exchange energies provides the missing link with the firstprinciples-based and electron-density-dependent effective embedding potential proposed recently. ${ }^{29}$ Finally, a discussion is given to provide explicit context for applications (and their possible limitations) to other systems.

\section{II.Theory and Computations}

II.1. The LFDFT for Complexes of $\mathbf{f}$ Elements. II.1.1. The Computational Scheme. The energy levels of a $\mathrm{f}^{n}$ ion in a complex are described by the empirical Hamiltonian

$$
\mathbf{H}=\mathbf{H}_{\mathrm{ER}}+\mathbf{H}_{\mathrm{SO}}+\mathbf{H}_{\mathrm{LF}}
$$

where the three terms represent the effect of interelectron repulsion, spin-orbit coupling, and ligand field, respectively. In LF theory, $\mathbf{H}$ is represented on the basis of the 14f spin-orbitals as oneelectron integrals $\left(\mathbf{H}_{\mathbf{S O}}\right.$ and $\left.\mathbf{H}_{\mathbf{L F}}\right)$ and two-electron integrals $\left(\mathbf{H}_{\mathbf{E R}}\right)$. The matrix elements of $\mathbf{H}_{\mathbf{E R}}, \mathbf{H}_{\mathbf{S O}}$, and $\mathbf{H}_{\mathbf{L F}}$ are then calculated within the basis of the $\left(\begin{array}{c}14 \\ n\end{array}\right)$ Slater determinants (SD) $\boldsymbol{\Phi}_{\mu}=\mid \chi_{1} \chi_{2}$ $\ldots \chi_{n} \mid$, where $\chi_{i}=\left\{f_{-3}^{\beta} f_{-3}^{\alpha} \ldots f_{3}^{\alpha}\right\}$, using the well-known Slater rules and expressed in terms of a small number of model parameters to be determined from energies of electronic transitions in experiment. These are the Coulomb repulsion (Racah $R_{n}(n=0,1,2,3)$ or Slater-Condon $\left.\left(F_{k}, k=0,2,4,6\right)\right)$, the spin-orbit coupling constant $\zeta$, and the (in general 28 nonzero) parameters of the $7 \times$ 7 LF matrix. The LFDFT procedure of deriving all these parameters consists of the following steps: (i) a spin-restricted KSDFT-SCF calculation on the average-of-configuration (AOC) $\mathrm{f}^{n}$ (with a $n / 7$ occupation of each orbital) is carried out. In doing so, we ensure a good starting approximation with regards to a good statistical sampling of the full $\mathrm{f}^{n}$ manifold. In fact, we comply with the spherical symmetry inherent to LFT when approximating matrix elements of interelectronic repulsion through atomic electrostatic parameters, e.g., Racah or Slater-Condon. We thus assign any energetic effect due to deviation of the LF orbitals from the spherical symmetry to contributions to the one-electron, LF matrix. This recipe is also consistent with the prerequisites of the LF approach, where orbital relaxation is only taken into account at the level of averaging the electron density to provide proper LF orbitals, while all SD energies for later LF treatment are calculated without SCF iteration. (ii) Using the Kohn-Sham orbitals from the first step, the energies of all SD are calculated; finally, the SD energies are utilized (using a script, written in MATLAB ${ }^{30}$ ) to obtain all needed model parameters in a least-squares fit. There is a remarkable consistency between the energies of SD calculated using this LF parametrization and their DFT values calculated directly-standard deviations between two sets of data usually do not exceed $0.1 \mathrm{eV}$. The parameters are used then in a full CI ligand field program to calculate energies and electronic properties of all multiplets split out of a $\mathrm{d}^{n}$ or a $\mathrm{f}^{n}$ configuration. In this work we focus on the $7 \times$ 7 LF matrix, which takes a particularly simple form in the case of $O_{h}$ symmetry (see below). We have shown ${ }^{21 b}$ that the matrix of

(28) (a) Ballhausen, C. J.; Dahl, J. P. Theor. Chim. Acta (Berlin) 1974, 34, 169. (b) Ballhausen, C. J. Molecular Electronic Structures of Transition Metal Complexes; McGraw-Hill International Book Company: New York, 1979; pp 53-54.

(29) (a) Wesolowski, T. A.; Warshel, A. J. Phys. Chem. 1993, 97, 8050. (b) Zbiri, M.; Atanasov, M.; Daul, C. A.; Garcia-Lastra, J. M.; Wesolowski, T. A. Chem. Phys. Lett. 2004, 397, 441.

(30) MATLAB 6.1 scripts and programs can be obtained from the authors on request. 
the LF, resulting from the many electron treatment, is essentially the same (differences between two sets of data not exceeding $1-2 \%)$ as the one obtained using the following simple recipe.

Let us denote KS orbitals dominated by f functions which result from an AOC f $\mathrm{f}^{n}$ KSDFT-SCF calculation with column vectors $\vec{V}_{i}$ and their energies by $\epsilon_{i}^{\mathrm{KS}}$, the latter defining the diagonal matrix E. From the components of the eigenvector matrix built up from such columns one takes only the components corresponding to the $\mathrm{f}$ functions. Let us denote the square matrix composed of these column vectors by $\mathbf{U}$ and introduce the overlap matrix $\mathbf{S}$ :

$$
\mathbf{S}=\mathbf{U}^{\mathrm{T}} \cdot \mathbf{U}
$$

Since $\mathbf{U}$ is in general not orthogonal, we use Löwdin's symmetric orthogonalization scheme to obtain an equivalent set of orthogonal eigenvectors $(\mathbf{C})$ :

$$
\mathbf{C}=\mathbf{U} \cdot \mathbf{S}^{-1 / 2}
$$

We identify now these vectors with the eigenfunctions of the effective LF Hamiltonian $h_{\mathrm{LF}}^{\text {eff }}$ we seek as

$$
\varphi_{\mathrm{i}}=\sum_{\mu=1}^{7} c_{\mu i} f_{\mu} \quad(i=1-7)
$$

and the corresponding eigenvalues $\left\langle\varphi_{i}\left|h_{\mathrm{LF}}^{\mathrm{eff}}\right| \varphi_{i}\right\rangle$ with $\epsilon_{i}^{\mathrm{KS}}$. The $7 \times 7$ LF matrix $\mathbf{V}_{\mathbf{L F}}=\left\{h_{\mu v}\right\}$ is given by

$$
\mathbf{V}_{\mathbf{L F}}=\mathbf{C} \cdot \mathbf{E} \cdot \mathbf{C}^{\mathrm{T}}=\left\{h_{\mu \nu}\right\}=\left\{\sum_{i=1}^{7} c_{\mu i} i_{i}^{\mathrm{KS}} c_{\mathrm{v} i}\right\}
$$

Remarkably, the matrix $\mathbf{V}_{\mathbf{L F}}$ is obtained in a general form without any assumptions (such as is done in CFT or the AOM). It is particularly suited in cases of low symmetry and complex coordination geometries where application of CFT or AOM, because of the large number of model parameters, is not easy.

II.1.2. Analysis of the Contributions to the Orbital Energies from LFDFT: The BLDFT and CFDFT Models. The procedure described in the previous section allows us to calculate the matrix elements of $\mathbf{V}_{\mathbf{L F}}$ from KSDFT and thus to study the effect of the coordination geometry on the energy levels of the Ln complex. When forming a complex, the orbital levels of Ln undergo different perturbation from the ligands, which we intend to analyze more closely in sections III.3 and III.4. Quantitatively, orbital levels of a TM become affected by different terms in the one-electron Hamiltonian. First of all, nonorthogonality of metal and ligand orbitals has to be taken into account. As has been shown by Ballhausen and Dahl, ${ }^{28}$ orthogonalizing metal and ligand orbitals leads to a component of the orbital energy which, in first approximation, scales as the square of the group overlap integral. It is equivalent to the Pauli(exchange) repulsion exerted on the metal $\mathrm{f}$ or $\mathrm{d}$ electrons by the closed electron shells of the ligand. The larger the metal-ligand overlap is, the stronger is the repulsion. Thus, TM orbitals which have a stronger overlap with ligand orbitals undergo a larger destabilization by coordinated ligands than those which have a weaker overlap. In addition to the Pauli repulsion, a destabilizing effect by Coulomb repulsion terms due to the ligand electrons takes place. This is analogous (but not identical) to crystal field perturbations, the latter being accounted for by a peculiar form of the Coulomb potential (i.e., approximating coordinating ligand nuclei and electrons by point charges, or point dipoles). Finally, electron charge transfer from the ligand to the metal orbitals (in the case of $\sigma$ or $\pi$ donation) or vice versa (in the case of $\pi$ back-donation) can take place. In a first approximation, it also depends on the square of the group overlap integral, as postulated in the AOM. In the case of antibonding $\mathrm{d}$ electrons it has been interpreted, as due to an increase of kinetic energy in the bond region by Jørgensen. ${ }^{31,32}$ In some more mathematical terms, we used the following recipe which allows us to explore for the first time the role of the various terms in $\mathbf{V}_{\mathbf{L F}}$ using KSDFT.

The SCF procedure within the KS DFT yields a diagonal eigenvalue matrix $\mathbf{E}$ and a matrix of eigenvectors (columns) $\mathbf{C}$ which diagonalize the KS eigenproblem (eq 6). Within the basis of symmetry adapted, nonorthogonal basis functions, the latter is given by eq 6 . From $\mathbf{E}$ and $\mathbf{C}$ one can reconstruct the initial KS Hamiltonian using either

$$
\mathbf{H} \cdot \mathbf{C}=\mathbf{S} \cdot \mathbf{C} \cdot \mathbf{E}
$$

an orthogonal or a nonorthogonal basis set. Taking the first alternative, eq 6 can be rewritten in the form of eqs 7 or 8 , where $\mathbf{H}^{\prime}$ and $\mathbf{C}^{\prime}$ are defined by eqs 9 and 10 ,

$$
\begin{gathered}
\left(\mathbf{S}^{-1 / 2} \cdot \mathbf{H} \cdot \mathbf{S}^{-1 / 2}\right) \cdot\left(\mathbf{S}^{1 / 2} \cdot \mathbf{C}\right)=\left(\mathbf{S}^{1 / 2} \cdot \mathbf{C}\right) \cdot \mathbf{E} \\
\mathbf{H}^{\prime} \cdot \mathbf{C}^{\prime}=\mathbf{C}^{\prime} \cdot \mathbf{E} \\
\mathbf{H}^{\prime}=\mathbf{S}^{-1 / 2} \cdot \mathbf{H} \cdot \mathbf{S}^{-1 / 2} \\
\mathbf{C}^{\prime}=\mathbf{S}^{1 / 2} \cdot \mathbf{C}
\end{gathered}
$$

respectively. Thus, within the orthogonal basis one obtains

$$
\mathbf{H}^{\prime}=\mathbf{C}^{\prime} \cdot \mathbf{E} \cdot \mathbf{C}^{\prime \mathrm{T}}=\left[\begin{array}{ll}
\mathbf{H}_{\mathrm{ff}}^{\prime} & \mathbf{H}_{\mathrm{fp}}^{\prime} \\
\mathbf{H}_{\mathrm{pf}}^{\prime} & \mathbf{H}_{\mathrm{pp}}^{\prime}
\end{array}\right]
$$

Neglecting ligand-to-metal charge transfer $\left(\mathbf{H}_{\mathrm{fp}}^{\prime}\right)$, we focus now on $\mathbf{H}_{\mathrm{ff}}^{\prime}$. As has been discussed in detail by Ballhausen and Dahl, ${ }^{28}$ eigenvalues resulting from diagonalization of $\mathbf{H}_{\mathrm{ff}}^{\prime}$ account both for Coulombic (crystal-field-like) and for Pauli (exchange) repulsions. The latter contributions are connected with the overlap between the metal and the ligand orbitals, leading to the repulsive energy component of the LF just discussed. It is the representation of eqs 7-11 which ensures now metal-ligand orthogonality and allows us to write down the $\mathbf{V}_{\mathbf{L F}}$ in terms of a pseudopotential. ${ }^{28}$ This being extracted from a KSDFT procedure, we call this LF-model Ballhausen DFT (BLDFT). The effect of the exchange repulsion term can be excluded if one goes from the orthogonal to the nonorthogonal basis, thus getting the original form of $\mathbf{H}$ back (eq 6). This is given simply by

$$
\mathbf{H}=\mathbf{S}^{1 / 2} \cdot \mathbf{H}^{\prime} \cdot \mathbf{S}^{1 / 2}=\left[\begin{array}{ll}
\mathbf{H}_{\mathrm{ff}} & \mathbf{H}_{\mathrm{fp}} \\
\mathbf{H}_{\mathrm{pf}} & \mathbf{H}_{\mathrm{pp}}
\end{array}\right]
$$

Diagonalization of the submatrix $\mathbf{H}_{\mathrm{ff}}$ yields now eigenvalues affected solely by the Coulombic contributions; we denote this model by crystal field DFT(CFDFT).

II.2. One-Electron Levels of Rare Earth Ions in Octahedral Ligand Fields. In octahedral coordination, the f orbitals give rise to $\mathrm{a}_{2 \mathrm{u}}, \mathrm{t}_{2 \mathrm{u}}$, and $\mathrm{t}_{1 \mathrm{u}}$ irreducible representations and combine with ligand orbitals as shown in Figure 1. While the $\mathrm{a}_{2 \mathrm{u}}$ orbital has no counterparts at the ligand and is thus nonbonding, $\mathrm{t}_{2 \mathrm{u}}$ and $\mathrm{t}_{1 \mathrm{u}}$ orbitals form respectively $\pi$ and $\sigma+\pi$ bonds with ligand functions. f-orbital energies within the AOM are quantified using parameters of $\sigma$ and $\pi$ antibonding character, $e_{\sigma}, e_{\pi}$, or alternatively, in terms of the symmetry-independent parameters $\Delta_{1}$ and $\Delta_{2}$ (Figure 2), from which

(31) (a) Jørgensen, C. K. Chem. Phys. Lett. 1967, 1, 11. (b) Jørgensen, C. K.; Faucher, M.; Garcia, D. Chem. Phys. Lett. 1986, 128, 250.

(32) Jørgensen, C. K. Modern Aspects of Ligand Field Theory; NorthHolland Publishing Company: Amsterdam, 1971. 
Atanasov et al.
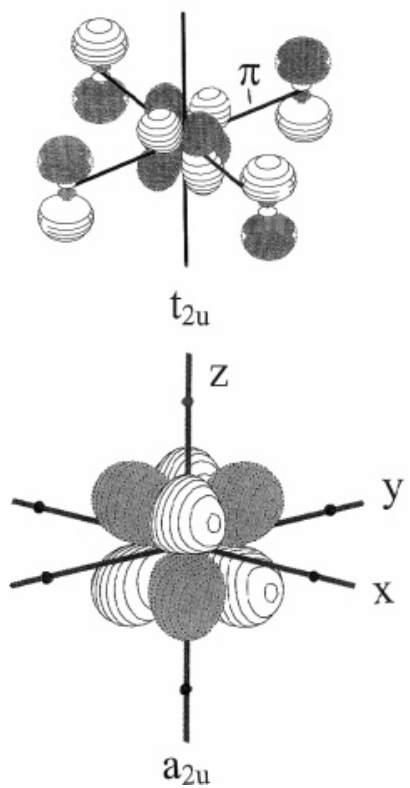

Figure 1. The $\mathrm{MO}$ orbitals of $4 \mathrm{f}$ type in an octahedral $\mathrm{YbCl}_{6}{ }^{3-}$ complex and the nonbonding $\mathrm{t}_{\mathrm{gg}}$ orbital of $\mathrm{Cl}$; only $\varsigma$ and $z$ components of the triply degenerate orbitals $t_{2 u}$ and $t_{1_{u}}, t_{1, g}$, respectively (see Supporting Information for their analytic definitions) are shown. Ligands without contributions to the nonbonding $\mathrm{a}_{2 \mathrm{u}}$ orbital are indicated by black points; orbital contours have been plotted for an electron density value of 0.05 chosen to show more clearly contributions from the $\mathrm{Cl} 3 \mathrm{p}$ and (for $\mathrm{t}_{1 \mathrm{u}}$ ) $3 \mathrm{~s}$ orbitals.

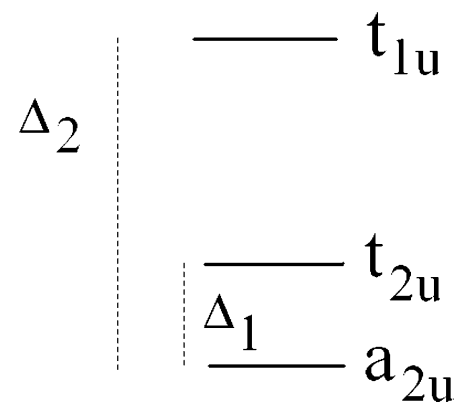

Figure 2. Symmetry-independent ligand field splitting parameters $\Delta_{1}$ and $\Delta_{2}$ for $\mathrm{f}$ orbitals in octahedral coordination.

the former can be calculated (see Supporting Information) utilizing eq 13.

$$
\begin{gathered}
e\left(\mathrm{a}_{2 \mathrm{u}}\right)=0 \\
\Delta_{1}=e\left(\mathrm{t}_{2 \mathrm{u}}\right)-e\left(\mathrm{a}_{2 \mathrm{u}}\right)=\frac{5}{2} e_{\pi} \\
\Delta_{2}=e\left(\mathrm{t}_{1 \mathrm{u}}\right)-e\left(\mathrm{a}_{2 \mathrm{u}}\right)=2 e_{\sigma}+\frac{3}{2} e_{\pi}
\end{gathered}
$$

For Ln ions, spin-orbit coupling dominates over the LF interaction and is particularly large for the latter members of the $4 \mathrm{f}$ series. It leads to a splitting of the $t_{2 u}$ and $t_{1 u}$ orbitals to give species of $\Gamma_{7}+\Gamma_{8}$ and $\Gamma_{6}+\Gamma_{8}$ symmetry, ${ }^{33}$ respectively, in the double group $\mathrm{O}^{*}$ and to $\Gamma_{7}$ for $\mathrm{a}_{2 \mathrm{u}}$ symmetry. As can be derived (cf. Supporting Information), the combined effect of spin-orbit coupling and ligand field is described by eqs 14 , yielding after diagonalization the energies of the one-electron states $2 \Gamma_{7}, 2 \Gamma_{8}$, and $\Gamma_{6}$ (for states with one hole signs in front of $\Delta_{1}, \Delta_{2}$, and $\varsigma$, the

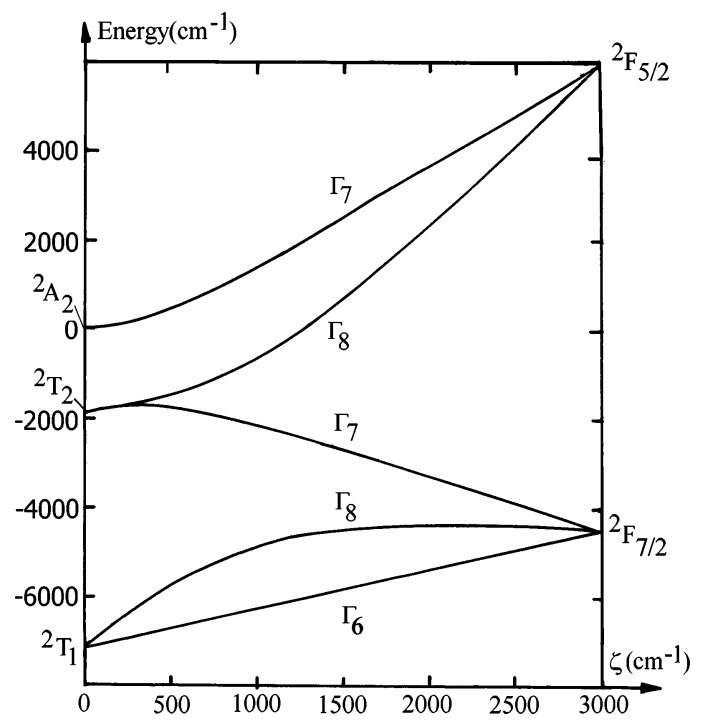

Figure 3. Energy level diagram dependent on the spin-orbit coupling constant $(\varsigma)$ for a $\mathrm{f}^{13} \mathrm{Ln}$ in octahedral coordination; in plotting this diagram values for $e_{\sigma}+\varsigma$ and $e_{\pi} / e_{\sigma}$ have been arbitrarily fixed at $3000 \mathrm{~cm}^{-1}$ and 0.25 , respectively.

spin-orbit constant, have to be correspondingly changed). A correlation energy diagram relating one-electron levels in the single (O) and double $\mathrm{O}^{*}$ group symmetries is presented in Figure 3 . It illustrates nicely the correlation of the one-electron levels in the limit of zero spin-orbit coupling $\left({ }^{2} \mathrm{~A}_{2},{ }^{2} \mathrm{~T}_{2}\right.$, and $\left.{ }^{2} \mathrm{~T}_{1}\right)$ with the ${ }^{2} \mathrm{~F}_{5 / 2}$ and ${ }^{2} \mathrm{~F}_{7 / 2}$ levels of the free $\mathrm{Yb}^{3+}$ ion (we take $e_{\sigma}+\varsigma=3000$ and $e_{\pi}=e_{\sigma} / 4$ and a variable $\varsigma$ ).

$$
\boldsymbol{\Gamma}_{6}: \frac{3}{2} \varsigma+\Delta_{2}
$$

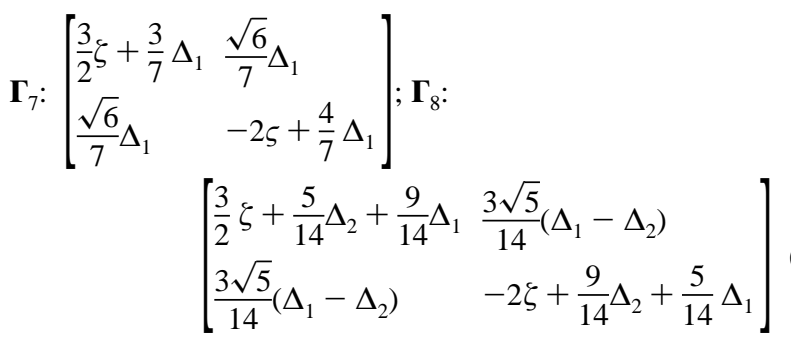

II.3. Computational Details. KSDFT calculations have been done using the ADF program package (program release ADF2003.01). ${ }^{34 a-d}$ The approximate Kohn-Sahm one-electron equations are solved by employing an expansion of the molecular orbitals based on Slater-type orbitals (STO). The PW91 exchange-correlation functional of the generalized gradient approximation type $\mathrm{e}^{35,36}$

(33) Different notations for the irreducible representations have been used in the literature. Here we adopt the notations by Bethe $\Gamma_{6}, \Gamma_{7}, \Gamma_{8}$. Alternative notations in the literature are those by Griffith, J. S.: $E^{\prime}$, $\mathrm{U}^{\prime}, \mathrm{E}^{\prime \prime}$ (Griffith, J. S. The theory of transition-metal ions; Cambridge University Press: Cambridge, 1971). Atkins, P. W.; Child, M. S.; Phillips, C. S. G. Tables for Group Theory; Oxford University Press: Oxford, 1984: $E_{1 / 2}, G_{3 / 2}, E_{5 / 2}$ and the ADF program package: $E_{1 / 2}$ $\mathrm{U}_{3 / 2}$, and $\mathrm{E}_{5 / 2}$, respectively.

(34) (a) Baerends, E. J.; Ellis, D. E.; Ros, P. Chem. Phys. 1993, 2, 42. (b) Boerrigter, P. M.; te Velde, G.; Baerends, E. J. Int. J. Quantum Chem. 1988, 33, 87. (c) teVelde, G.; Baerends, E. J. Comput. Phys. 1992 99, 84. (d) te Velde, G.; Bickelhaupt, F. M.; Baerends, E. J.; Fonesca Guerra, C.; van Gisbergen, S. J. A.; Snijders, J. G.; Ziegler, T. J. Comput. Chem. 2001, 22, 931-967 and cited references. (e) van Lenthe, E.; Baerends, E. J.; Snijders, J. G. J. Chem. Phys. 1993, 99 , 4597.

(35) ADF Users manual and cited references, http://www.scm.com. 


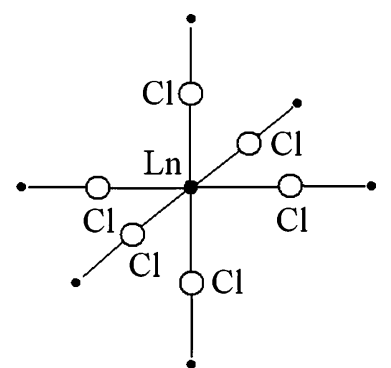

Figure 4. Model cluster for DFT-based ligand field calculations of $\mathrm{LnCl}_{6}{ }^{3-}$ complexes.

Table 1. $\mathrm{Ln}-\mathrm{Cl}$ Distances (in $\AA$ ), $\mathrm{f}^{n}$ Configurations and the Occupation Numbers for the $\mathrm{f}$ Orbitals Adopted in LFDFT Calculations of $\mathrm{LnCl}_{6}{ }^{3-}$ Model Clusters and $\mathrm{Ln}^{3+}$ in a Point Charge Environment Representing the $\mathrm{Cl}^{-}$Ligands and Nearest Neighbor $\mathrm{Na}^{+}$Ions ${ }^{a}$

\begin{tabular}{lccccc}
\hline $\mathrm{Ln}$ & $R$ & $\mathrm{f}^{n}$ & $\mathrm{a}_{2 \mathrm{u}}$ & $\mathrm{t}_{2 \mathrm{u}}$ & $\mathrm{t}_{1 \mathrm{u}}$ \\
\hline $\mathrm{Ce}$ & 2.820 & $\mathrm{f}^{1}$ & 0.143 & 0.428 & 0.429 \\
$\mathrm{Pr}$ & 2.800 & $\mathrm{f}^{2}$ & 0.286 & 0.857 & 0.857 \\
$\mathrm{Nd}$ & 2.793 & $\mathrm{f}^{3}$ & 0.428 & 1.286 & 1.286 \\
$\mathrm{Sm}$ & 2.768 & $\mathrm{f}^{5}$ & 0.714 & 2.143 & 2.143 \\
$\mathrm{Eu}$ & 2.760 & $\mathrm{f}^{6}$ & 0.857 & 2.571 & 2.572 \\
$\mathrm{~Tb}$ & 2.733 & $\mathrm{f}^{8}$ & 1.143 & 3.428 & 3.429 \\
$\mathrm{Dy}$ & 2.722 & $\mathrm{f}^{9}$ & 1.286 & 3.857 & 3.857 \\
$\mathrm{Ho}$ & 2.711 & $\mathrm{f}^{10}$ & 1.428 & 4.286 & 4.286 \\
$\mathrm{Er}$ & 2.700 & $\mathrm{f}^{11}$ & 1.571 & 4.714 & 4.715 \\
$\mathrm{Tm}$ & 2.690 & $\mathrm{f}^{12}$ & 1.714 & 5.143 & 5.143 \\
$\mathrm{Yb}$ & 2.680 & $\mathrm{f}^{13}$ & 1.857 & 5.571 & 5.571
\end{tabular}

${ }^{a}$ Not listed are the fully occupied MOs $\mathrm{a}_{1 \mathrm{~g}}{ }^{20} \mathrm{eg}^{28} \mathrm{t}_{1 \mathrm{~g}}{ }^{12} \mathrm{t}_{2 \mathrm{~g}}{ }^{24} \mathrm{t}_{2 \mathrm{u}}{ }^{12} \mathrm{t}_{1 \mathrm{u}}{ }^{66}$ (for $\mathrm{LnCl}_{6}{ }^{3-}$ ) and $\mathrm{a}_{1 \mathrm{~g}}{ }^{10} \mathrm{eg}_{\mathrm{g}} \mathrm{t}_{2 \mathrm{~g}} \mathrm{~g}^{12} \mathrm{t}_{1 \mathrm{u}}{ }^{24}$ (for $\mathrm{Ln}^{3+}$ in a point charge environment).

was used in all KSDFT calculations. Scalar relativistic calculations have been done within the Zero-Order Regular Approximation ${ }^{34 \mathrm{e}}$ (ZORA) adopting appropriate (ZORA) all-electron triple- $\varsigma$ STO basis sets for $\mathrm{Ln}$ and $\mathrm{Cl}$ (TZP). To study crystal field contributions to the splitting of the $\mathrm{f}$ orbitals, model calculations have been made in which atoms surrounding Ln have been approximated by point charges. Increasing default values for numerical integration and point charges (ADF input: accint 8.0 and qpnear 10) in this case allowed more accurate results to be obtained and compared with full calculations on $\mathrm{LnCl}_{6}{ }^{3-}$.

In this study we consider energy levels and ligand fields deduced from spectra of cubic elpasolites $\mathrm{Cs}_{2} \mathrm{NaLnCl}_{6}$ and the $\mathrm{Cs}_{2} \mathrm{NaYCl}_{6}$ : $\mathrm{Ln}$ and their interpretations. To mimic the effect of $\mathrm{Na}^{+}$on $\mathrm{LnCl}_{6}{ }^{3-}$, we placed point charges $q$ behind the $\mathrm{Ln}-\mathrm{Cl}$ bonds (Figure 4) at a distance of $2.83 \AA$, which equals the $\mathrm{Na}-\mathrm{Cl}$ distance, and varied $q$ between zero (for the bare anion) to $q=+1$, the formal charge of $\mathrm{Na}^{+}$. As for the $\mathrm{Ln}-\mathrm{Cl}$ bond distances $R$, sums of ionic radii of the respective $\mathrm{Ln}^{3+}$ and $\mathrm{Cl}^{-}$have been used as given by the Tables of Shannon; ${ }^{37}$ values of $R$ and f-orbital occupancies are collected in Table 1.

\section{Results and Discussion}

III.1. Metal (4f) - Cl 3p Mixing from VBCI Analysis of $4 \mathrm{f} \rightarrow \mathbf{4 f}$ and $\mathrm{Cl} 3 \mathrm{p} \rightarrow \mathbf{4 f}$ Charge-Transfer Spectra. Optical spectra of neat $\mathrm{Cs}_{2} \mathrm{NaLnCl}_{6}$ and doped $\mathrm{Cs}_{2} \mathrm{NaYCl}_{6}: \mathrm{Ln}^{3+}$ systems have been studied and CF energy levels split out of the $4 \mathrm{f}^{n}$ electronic configuration of the Ln have been fully characterized. A valuable collection of spectral data on the $\mathrm{LnCl}_{6}{ }^{3-}$ chromophors with exact $O_{h}$ symmetry and their

(36) (a) Perdew, J. P.; Wang. Y. Phys. Rev. B 1986, 33, 8800. (b) Perdew, J. P.; Chevary, J. A.; Vosko, S. H.; Jackson, K. A.; Pederson, M. R.; Singh, D. J.; Fiolhais, C. Phys. Rev. B 1992, 46, 6671; Error correction: Phys. Rev. B 1993, 48, 4978.

(37) Shannon, R. D. Acta Crystallogr. A 1976, 32, 751.
Table 2. Crystal Field Parameters ( $B_{40}$ and $B_{60}$ from Ref 38 ), Their Equivalent Values within the Angular Overlap Model $e_{\sigma}$ and $e_{\pi}$ and the Spin-Orbit Coupling Constant $\varsigma$ (All Parameters in $\mathrm{cm}^{-1}$ ) for $\mathrm{LnCl}_{6}{ }^{3-}$ Chromophores, as Obtained from a Fit to Best Available Electronic Absorption Spectra

\begin{tabular}{lccccc}
\hline $\mathrm{Ln}$ & $B_{40}$ & $B_{60}$ & $e_{\sigma}$ & $e_{\pi}$ & \multicolumn{1}{c}{} \\
\hline $\mathrm{Ce}$ & 2120 & 258 & 419 & 156 & 623 \\
$\mathrm{Pr}$ & 2290 & 236 & 447 & 185 & 756 \\
$\mathrm{Nd}$ & 1966 & 258 & 391 & 137 & 872 \\
$\mathrm{Sm}$ & 1620 & 247 & 326 & 100 & 1167 \\
$\mathrm{Eu}$ & 1935 & 250 & 384 & 136 & 1324 \\
$\mathrm{~Tb}$ & 1628 & 148 & 315 & 140 & 1694 \\
$\mathrm{Dy}$ & 1558 & 130 & 300 & 138 & 1920 \\
$\mathrm{Ho}$ & 1678 & 153 & 325 & 143 & 2129 \\
$\mathrm{Er}$ & 1495 & 156 & 292 & 120 & 2356 \\
$\mathrm{Tm}$ & 1553 & 175 & 305 & 120 & 2624 \\
$\mathrm{Yb}$ & 1632 & 279 & 333 & 88 & 2866
\end{tabular}

interpretation ${ }^{38}$ allowed reliable values of CF parameters $B_{40}$ and $B_{60}$ and the spin-orbit coupling constant $\varsigma$ (Table 2) to be obtained.

$B_{40}$ and $B_{60}$ are parameters depending on the expectation values of $\left\langle r^{4}\right\rangle_{4 \mathrm{f}}$ and $\left\langle r^{6}\right\rangle_{4 \mathrm{f}}$ and on the $\mathrm{Ln}-\mathrm{Cl}$ bond distance $R$ via $R^{-5}$ and $R^{-7}$, respectively, thus determining the radial dependence of the nonspherically symmetric component of the CF potential ${ }^{39}$ (eq 15, $Y_{\mathrm{k}, \mathrm{m}}$, the spherical harmonics normalized to 1). However, being determined from experiment, these parameters reflect contributions from covalency as well. Thus, they can be regarded as experimental sources for the values of $e_{\sigma}$ and $e_{\pi}$ or, alternatively, for the parameters $\Delta_{1}$ and $\Delta_{2}$, allowing these to be obtained from the data listed in Table 2. To do this, we make use respectively of eq 13 and eq 16 (for derivation see Supporting Information). Values of $e_{\sigma}$ and $e_{\pi}$ and $\Delta_{1}$ and $\Delta_{2}$ are listed in Tables 2 and 3(last column), respectively.

$$
\begin{gathered}
H_{\mathrm{CF}}=B_{40}\left[C_{40}+\sqrt{\frac{5}{14}}\left(C_{44}+C_{4-4}\right)\right]+B_{60}\left[C_{60}-\right. \\
\sqrt{\frac{7}{2}}\left(C_{64}+C_{6-4}\right) \\
C_{\mathrm{km}}(\theta, \varphi)=\left(\frac{4 \pi}{2 k+1}\right)^{1 / 2} Y_{\mathrm{k}, \mathrm{m}}(\theta, \varphi) \\
\Delta_{1}=\frac{10}{33} B_{40}-\frac{140}{143} B_{60} \\
\Delta_{2}=\frac{6}{11} B_{40}-\frac{140}{429} B_{60}
\end{gathered}
$$

To the extent, following the $\mathrm{AOM}$, that $e_{\sigma}$ and $e_{\pi}$ can be interpreted as solely due to covalency (cf. section III.3), the VBCI model allows $e_{\lambda}(\lambda=\sigma, \pi)$ to be related to the transfer (hopping) integrals $t_{\mathrm{pf} \lambda}$ and the CT energy $\Delta_{\mathrm{CT}}$, i.e., the

(38) (a) Richardson, F. S.; Reid, M. F.; Dallara, J. J.; Smith, R. D. J. Chem. Phys. 1985, 83,$3813 ; B_{40}$ and $B_{60}$ in our work are those given by Wybourne; they are related to the data of this reference as given by $B_{40}=0.8865 B_{0}^{(4)} ; B_{60}=-0.783 B_{0}^{(6)} . B_{40}$ and $B_{60}$ are connected with $A_{40}\left\langle r^{4}\right\rangle$ and $A_{60}\left\langle r^{6}\right\rangle$ introduced by the operator equivalent technique as given by $B_{40}=8 A_{40}\left\langle r^{4}\right\rangle$ and $B_{60}=16 A_{60}\left\langle r^{6}\right\rangle$; they are also related to the quantities $f_{4}=\left\langle r^{4}\right\rangle / R^{5}$ and $f_{6}=\left\langle r^{6}\right\rangle / R^{7}$ as follows: $B_{40}=c \cdot(7 /$ $2) \cdot Z \cdot f_{4}$ and $B_{60}=c \cdot(3 / 4) \cdot Z \cdot f_{6}$ with $\left\langle r^{4}\right\rangle,\left\langle r^{6}\right\rangle$, and $R$ in $\AA$ and $B_{40}$ and $B_{60}$ in $\mathrm{cm}^{-1}, c$ conversion factor $(=116093)$. (b) Reid, M. F.; Richardson, F. S. J. Chem. Phys. 1985, 83, 3831.

(39) Sugano, S.; Tanabe, Y.; Kamimura, H. Multiplets of Transition-Metal Ions in Crystals; Academic Press: New York, 1970. In deriving eq 9 we made use of Table 1.2 in this book. 
Table 3. Ligand Field Splitting Parameters $\Delta_{1}, \Delta_{2}\left(\right.$ in $\left.\mathrm{cm}^{-1}\right)$ Calculated from a Fit to Best Available Energy Level Data of $\mathrm{LnCl}_{6}{ }^{3-}$ Chromophores in $\mathrm{Cs}_{2} \mathrm{NaLnCl}_{6}$ and Values of $\Delta_{1}, \Delta_{2}$ from Average-of-Configuration (AOC) KSDFT Calculations Utilizing the LFDFT, BLDFT, CFDFT, and CFDFT_pc Models ${ }^{a}$

\begin{tabular}{lrrrrr}
\hline & \multicolumn{5}{c}{$\Delta_{1}$} \\
& \multicolumn{5}{c}{$\Delta_{2}$} \\
\cline { 2 - 5 } & \multicolumn{5}{c}{ DFT } \\
\cline { 2 - 5 } Ln & LFDFT & BLDFT & CFDFT & CFDFT_pc & experiment $^{b}$ \\
\hline $\mathrm{Ce}$ & 331 & 288 & -266 & 265 & $390 \pm 1$ \\
& 874 & 1650 & -464 & 577 & $1072 \pm 1$ \\
$\mathrm{Pr}$ & 370 & 281 & -144 & 228 & $462 \pm 20$ \\
& 958 & 1401 & -250 & 492 & $1172 \pm 16$ \\
$\mathrm{Nd}$ & 410 & 266 & -76 & 200 & $343 \pm 41$ \\
& 1027 & 1235 & -102 & 428 & $988 \pm 34$ \\
$\mathrm{Sm}$ & 518 & 287 & 33 & 163 & $250 \pm 18$ \\
& 1194 & 1113 & 93 & 343 & $803 \pm 15$ \\
$\mathrm{Eu}$ & 566 & 295 & 71 & 150 & $341 \pm 24$ \\
& 1252 & 1048 & 176 & 314 & $973 \pm 19$ \\
$\mathrm{~Tb}$ & 664 & 281 & 94 & 131 & $349 \pm 17$ \\
& 1385 & 979 & 209 & 270 & $840 \pm 13$ \\
$\mathrm{Dy}$ & 723 & 289 & 119 & 118 & $345 \pm 26$ \\
& 1454 & 956 & 248 & 244 & $808 \pm 21$ \\
$\mathrm{Ho}$ & 806 & 282 & 124 & 116 & $358 \pm 29$ \\
& 1538 & 919 & 248 & 239 & $865 \pm 23$ \\
$\mathrm{Er}$ & 906 & 272 & 127 & 110 & $300 \pm 34$ \\
& 1632 & 894 & 257 & 226 & $764 \pm 29$ \\
$\mathrm{Tm}$ & 1089 & 237 & 104 & 102 & $299 \pm 27$ \\
& 1784 & 813 & 206 & 207 & $790 \pm 22$ \\
$\mathrm{Yb}$ & 1711 & 180 & 53 & 102 & $221 \pm 19$ \\
& 2248 & 712 & 151 & 206 & $799 \pm 34$
\end{tabular}

${ }^{a}$ DFT values that agree best with experiment are italicized. Average and maximal deviations of italicized values from experimental ones are as follows: BLDFT -52 and 76 (for $\Delta_{1}$ ) and 120 and 310 (for $\Delta_{2}$ ); LFDFT: 73 and 92 (for $\Delta_{1}$ ) and 150 and 214 (for $\Delta_{2}$ ). ${ }^{b}$ Deduced from the data collection in ref 38(a); error bars: from ref 38(b) and eq 16.

Table 4. First Charge Transfer Absorption Band Maxima $\left(\Delta_{\mathrm{CT}}\right)$ for Some $\mathrm{LnCl}_{6}{ }^{3-}$ Ions and Values of the Transfer (Hopping) Integrals $\mathrm{t}_{\mathrm{pf} \sigma}$ and $\mathrm{t}_{\mathrm{pf} \pi}\left(\mathrm{in}^{-1}\right)$ and Percentages of $4 \mathrm{f}(\mathrm{Ln})-3 \mathrm{p}(\mathrm{Cl})$ Mixing of $\sigma$ $(\% \sigma)$ and $\pi(\% \pi)$ Type Deduced Using the VBCI Model

\begin{tabular}{lrrrrr}
\hline complex & \multicolumn{1}{c}{$\Delta_{\mathrm{CT}^{a}}$} & $\mathrm{t}_{\mathrm{pf} \sigma}$ & $\mathrm{t}_{\mathrm{pf} \pi}$ & $\% \sigma$ & $\% \pi$ \\
\hline $\mathrm{SmCl}_{6}{ }^{3-}$ & 43100 & 3748 & 2076 & 0.8 & 0.2 \\
$\mathrm{EuCl}_{6}{ }^{3-}$ & 33200 & 3570 & 2125 & 1.2 & 0.4 \\
$\mathrm{TmCl}_{6}{ }^{3-}$ & $\approx 46800$ & 3778 & 2370 & 0.6 & 0.3 \\
$\mathrm{YbCl}_{6}{ }^{3-}$ & 38320 & 3572 & 1836 & 0.9 & 0.2
\end{tabular}

${ }^{a}$ From a comparison in ref 41 .

energy needed to transfer one electron from the nonbonding $\mathrm{t}_{1 \mathrm{~g}}$ orbital of $\mathrm{Cl}^{-}$(Figure 1) to the 4 f orbitals of the $\mathrm{Ln}^{3+}$ (eq 17). Experimental values of $\Delta_{\mathrm{CT}}$ obtained from CT spectra of selected members of $\mathrm{LnCl}_{6}{ }^{3-}$ are listed in Table 4. They allow calculation of $t_{\mathrm{pf} \lambda}$ as well as the extent of $3 \mathrm{p}(\mathrm{Cl})$ and $4 \mathrm{f}(\mathrm{Ln})$ mixing according to eq 18 . We thus arrive at estimates of the extent of $\mathrm{Ln}-\mathrm{Cl}$ covalency

$$
\begin{gathered}
e_{\lambda}=\frac{t_{\mathrm{pf} \lambda}^{2}}{\Delta_{\mathrm{CT}}} \quad(\lambda=\sigma, \pi) \\
\varphi_{\mathrm{KS}}=c_{1} \chi_{\mathrm{Ln}, 4 \mathrm{f}}+c_{2} \chi_{\mathrm{Cl}, 3 \mathrm{p}} ; c_{1}{ }^{2}+c_{2}{ }^{2} \approx 1 \\
\% \lambda \cong c_{2}{ }^{2} \cdot 100=\left(\frac{t_{\mathrm{pf} \lambda}}{\Delta_{\mathrm{CT}}}\right)^{2} \cdot 100
\end{gathered}
$$

on the basis of experimental data. Keeping in mind that the experimentally determined parameters $e_{\lambda}$ reflect contributions not only from covalency but also from Coulomb and exchange repulsion effects, it follows that the values of the mixing coefficients estimated in such a way are upper bounds for the extent of the ligand-to-metal charge transfer. Our results clearly show that $3 \mathrm{p}-4 \mathrm{f}$ mixing does not exceed $1-2 \%$ and is larger for $\sigma$ than for $\pi$ interactions, as expected. In section III. 2 we make use of this result in order to check the DFT calculations.

III.2. DFT Results. Metal-ligand covalency in complexes with predominant ionic bonding is governed by the admixture of ligand-to-metal charge-transfer excited states into the (ionic) ground-state wave function. Within the VBCI model this is well accounted for by two parameters and their ratio $t / \Delta_{\mathrm{CT}}$ (see above). Generally speaking, DFT is not able to yield parameters $t$ and $\Delta_{\mathrm{CT}}$ to be compared with semiempirical ones deduced from experiment because quantities provided by the DFT will possibly depend on the particular choice of KSDFT orbitals and the functional used in their calculation. Thus, any unitary transformation among the KS orbitals will not change the density and therefore the total electronic energy of the system. Yet canonical KS orbitals are found to have chemical significance ${ }^{40}$ and moreover are very useful in the sense that they are well suited for discussion of energies of electronic transitions in terms of orbital energy differences. Thus, from the difference between the energies of a pair of bonding (b)-antibonding (a) orbitals, $\Delta E=e(a)-e(b)$, and the MO coefficient $(c)$ of the metal (ligand) orbitals to $a(b)$, effective DFT parameters $\Delta_{\mathrm{CT}}^{\prime}$ and $t^{\prime}$ corresponding to the matrix

$$
\left[\begin{array}{cc}
0 & t^{\prime} \\
t^{\prime} & -\Delta_{\mathrm{CT}}^{\prime}
\end{array}\right]
$$

can be reconstructed using the equations

$$
\begin{gathered}
t^{\prime}=c \sqrt{1-c^{2}} \Delta E \\
\Delta_{\mathrm{CT}}^{\prime}=\left(2 c^{2}-1\right) \Delta E
\end{gathered}
$$

The MO energy diagram of $\mathrm{YbCl}_{6}{ }^{3-}$ surrounded by $6(+1)$ point charges (Figure 5) shows three bonding orbitals $10 \mathrm{a}_{1 \mathrm{~g}}$, $7 \mathrm{e}_{\mathrm{g}}$, and $10 \mathrm{t}_{1 \mathrm{u}}$ dominated by ligand functions as expected. For the sake of comparison, in Figure 5, we have also shown the $4 \mathrm{f}$ orbitals on $\mathrm{Yb}^{3+}$ and $3 \mathrm{p}$ orbitals on $\mathrm{Cl}_{6}{ }^{6-}$ affected by the electrostatic field of the remaining part of the cluster which we account for by formal point charges $(q=-1$ for $\mathrm{Cl}^{-},+1$ for $\mathrm{Na}^{+}$, and +3 for $\left.\mathrm{Yb}^{3+}\right)$. The $4 \mathrm{f}$ and $3 \mathrm{p}$ orbitals of the separate $\mathrm{Yb}^{3+}$ and $\mathrm{Cl}_{6}{ }^{6-}$ fragments are significantly split by $\mathrm{CF}$ (for $\mathrm{Yb}^{3+}$ ) and the combination of the $\mathrm{CF}$ and $\mathrm{Cl}-\mathrm{Cl}$ overlap (for $\mathrm{Cl}_{6}{ }^{6-}$ ). Due to the strong $\mathrm{Cl}-\mathrm{Cl}$ interactions in the latter case, $\mathrm{t}_{2 \mathrm{u}}$ and $\mathrm{t}_{1 \mathrm{u}} 3 \mathrm{p}$ orbitals of $\mathrm{Cl}$ become destabilized and approach closely the $4 \mathrm{f}$ orbitals of Yb. This is the cause for an artificially large mixing between 4f and $3 \mathrm{p}$ orbitals; thus, $\mathrm{Cl} 3 \mathrm{p}$ orbitals are calculated to contribute 47 and $36 \%$ to the $3 \mathrm{t}_{2 \mathrm{u}}$ and $12 \mathrm{t}_{1 \mathrm{u}}$ MOs, respec-

(40) (a) Kohn, W.; Becke, A. D.; Parr, R. G. J. Phys. Chem. 1996, 100, 12974. (b) Baerends, E. J.; Gritsenko, O. V. J. Phys. Chem. A 1997, 101, 5383. (c) Stowasser, R.; Hoffmann, R. J. Am. Chem. Soc. 1999, 121, 3414. (d) Baerends, E. J. Theor. Chem. Acc. 2000, 103, 265.

(41) Nugent, L. J.; Baybarz, R. D.; Burnett, J. L.; Ryan, J. L. J. Phys. Chem. 1973, 77, 1528. 


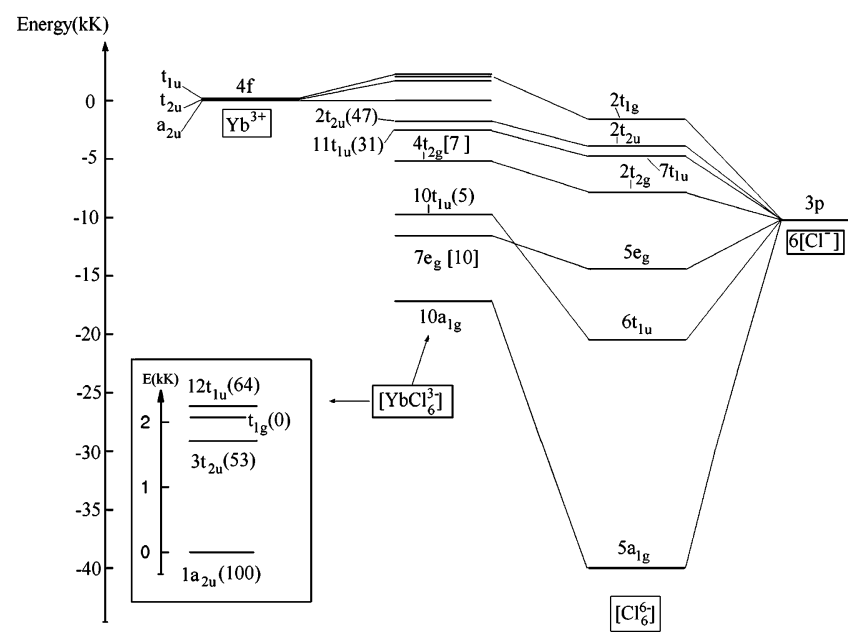

Figure 5. Orbital energies (in $\mathrm{kK}, 1 \mathrm{kK}=1000 \mathrm{~cm}^{-1}$ ) from KS-DFT calculation of the charge-compensated $\mathrm{YbCl}_{6}{ }^{3-}$ complex (middle and insert) and of the constituent $\mathrm{Yb}^{3+}$ (left) and $\mathrm{Cl}_{6}{ }^{6-}$ (right) fragments in an environment of $6(-1)+6(+1)$ and $(3+)+6(+1)$ point charges, respectively, completing the coordination sphere in the solid. The position of the $3 \mathrm{p}$ orbitals for six noninteracting $\mathrm{Cl}^{-}$ions is also given. The splitting of the $\mathrm{f}$ orbitals and the position of the nonbonding $2 \mathrm{t}_{1 \mathrm{~g}}$ orbital of the $\mathrm{Cl}_{6}{ }^{6-}$ fragment are more clearly shown in the insert. Percentages of $4 \mathrm{f}$ and $5 \mathrm{~d}$ orbitals of $\mathrm{Yb}$ to the MOs of $\mathrm{YbCl}_{6}{ }^{3-}$ are listed by normal and square brackets, respectively. Contributions from the $6 \mathrm{~s}$ and $6 \mathrm{p}$ orbitals of $\mathrm{Yb}$ to the $\mathrm{a}_{1 \mathrm{~g}}$ and $\mathrm{t}_{1 \mathrm{u}}$ MOs do not exceed $3 \%$ and are not listed.

Table 5. Effect of Compensating Point Charges $(q)$ on the LFDFT Splitting Parameters $\Delta_{1}$ and $\Delta_{2}$, Their AOM Representation in Terms of $e_{\sigma}$ and $e_{\pi}$ (All in $\mathrm{cm}^{-1}$ ), and the $4 \mathrm{f}$ Percentage Contributions to the LF Orbitals $\mathrm{t}_{2 \mathrm{u}}$ and $\mathrm{t}_{1 \mathrm{u}}$ for $\mathrm{CeCl}_{6}{ }^{3-}$ and $\mathrm{YbCl}_{6}{ }^{3-}$

\begin{tabular}{|c|c|c|c|c|c|c|c|}
\hline & & $\Delta_{1}$ & $\Delta_{2}$ & $e_{\sigma}$ & $e_{\pi}$ & $\% f$ in $t_{2 u}$ & $\% \mathrm{f}_{\text {in }} \mathrm{t}_{1 \mathrm{u}}$ \\
\hline $\mathrm{Ce}$ & $\begin{array}{l}q=0 \\
q=1\end{array}$ & $\begin{array}{l}330 \\
331\end{array}$ & $\begin{array}{r}1030 \\
874\end{array}$ & $\begin{array}{l}416 \\
338\end{array}$ & $\begin{array}{l}132 \\
132\end{array}$ & $\begin{array}{l}98.7 \\
98.6\end{array}$ & $\begin{array}{l}96.5 \\
96.7\end{array}$ \\
\hline $\mathrm{Yb}$ & $\begin{array}{l}q=0 \\
q=1\end{array}$ & $\begin{array}{l}1467 \\
1711\end{array}$ & $\begin{array}{l}2692 \\
2248\end{array}$ & $\begin{array}{l}906 \\
611\end{array}$ & $\begin{array}{l}587 \\
684\end{array}$ & $\begin{array}{l}63.6 \\
53.1\end{array}$ & $\begin{array}{l}61.2 \\
63.5\end{array}$ \\
\hline
\end{tabular}

tively. According to the results in section III.1, these orbitals should be dominated by not less than $99 \%$ by $4 \mathrm{f}$ contributions. This is clearly an artifact connected with the anomalously large energy of the $\mathrm{Cl}-3 \mathrm{p}$ orbitals, falling in the same range as the $4 \mathrm{f}$ orbitals (see insert in Figure 5). Thus, application of eqs 19 and 20 to the set of the bonding (respectively antibonding) $\mathrm{t}_{2 \mathrm{u}}$ orbital, a value for $t_{\mathrm{pf} \pi}$ is obtained $\left(1718 \mathrm{~cm}^{-1}\right)$, which matches closely the one deduced from VBCI and experiment $\left(1836 \mathrm{~cm}^{-1}\right)$. However, $\Delta_{\mathrm{CT}}^{\prime}$ is nearly vanishing! Because of this, LFDFT values of $\Delta_{1}$ and $\Delta_{2}$ (1711 and $2248 \mathrm{~cm}^{-1}$ ) exceed the ones deduced from experiment by factors of 8 and 3, respectively (cf. last column of Table 3 ). This result does not change significantly, if instead of adding 6 counter charges with $q=1$ in the calculations of the bare $\mathrm{YbCl}_{6}{ }^{3-}$ anion $q$ is set to zero (Table 5). When going from $q=1$ to $q=0, \Delta_{1}$ and $\Delta_{2}$ decrease and increase, respectively, by 14 and $20 \%$, but the discrepancy with experiment remains. A rather different result is obtained for $\mathrm{CeCl}_{6}{ }^{3-}$ (Table 5); here, the $4 \mathrm{f}$ functions of $\mathrm{t}_{2 \mathrm{u}}$ and $\mathrm{t}_{\mathrm{lu}}$ symmetry for $\mathrm{Ce}^{3+}$ are almost pure metal functions. In accord with this, LFDFT values of $\Delta_{1}$ and $\Delta_{2}$ (331 and $874 \mathrm{~cm}^{-1}$ ) compare reasonably to the ones deduced from experiment (390 and $1072 \mathrm{~cm}^{-1}$, cf. Table 3). Again, results do not change significantly if the bare anion, instead of the charge-compensated one, is considered (Table 5); $\Delta_{1}$ is

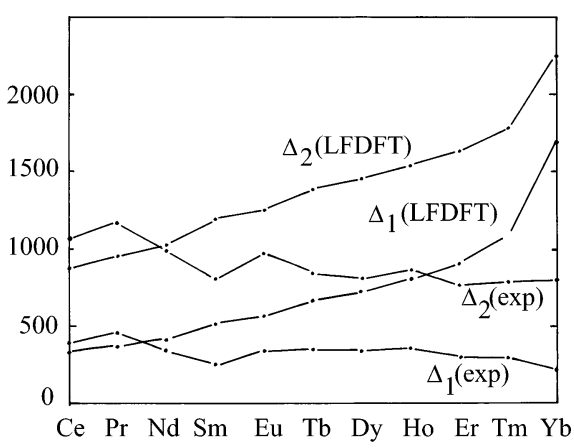

Figure 6. Values of $\Delta_{1}$ and $\Delta_{2}$ (in $\mathrm{cm}^{-1}$ ) from LFDFT calculations and from the interpretation of the $\mathrm{f}-\mathrm{f}$ spectra (experiment, ref 38 ) for $\mathrm{LnCl}_{6}{ }^{3-}$ complexes.

almost constant, but $\Delta_{2}$ increases (as in the case of $\mathrm{Yb}^{3+}$ ) by $18 \%$. These changes are best interpreted in terms of the AOM parameters $e_{\sigma}$ and $e_{\pi}$ (Table 5). Placing a positive charge behind each $\mathrm{Ce}-\mathrm{Cl}$ bond correlates with a decrease of the value of $e_{\sigma}$, while leaving $e_{\pi}$ essentially unchanged. This is also intuitively expected on the basis of the polarization by the positive point charge of the $3 \mathrm{p}_{\sigma}$ orbitals of $\mathrm{Cl}$ and its weaker effect on the $3 \mathrm{p}_{\pi}$ orbitals.

III.3. A Procedure of Eliminating Artificial $4 \mathbf{f}-\mathbf{3 p}$ Charge Transfer for Latter Ln Complexes in DFT. The comparison of the LFDFT values for $\Delta_{1}$ and $\Delta_{2}$ with the experimental ones (Table 3, Figure 6) clearly demonstrates that the two sets of data deviate increasingly from each other when going from left to right in the series, the discrepancy being largest for $\mathrm{Yb}^{3+}$. While experiment does not show any significant variation of $\Delta_{1}$ and $\Delta_{2}$ across the series, $\Delta_{1}$ and $\Delta_{2}$ values obtained from LFDFT increase from left to right of the series. Is it possible to cure this situation within the KSDFT framework?

$\Delta_{1}$ and $\Delta_{2}$ from LFDFT calculations can be decomposed into three different terms: Pauli(exchange) repulsion, Coulombic (CF-like) repulsion, and ligand-to-metal charge transfer (see section II.1.2). The effect of ligand-to-metal charge transfer can be eliminated in two different ways, thus allowing contributions from Pauli and Coulomb repulsion or the pure Coulombic effects to be obtained separately. These are given by the BLDFT and CFDFT models, respectively, which utilize metal orbitals which have been orthogonalized (BLDFT) or not (CFDFT) with respect to the ligand basis functions (see section II.1.2). Finally, one can discuss LF splitting approximating ligands and further electrostatic surroundings by a model potential of point charges. We denote this DFT model by CFDFT_pc. In Table 3 values of $\Delta_{1}$ and $\Delta_{2}$ obtained from LFDFT, BLDFT, CFDFT, and CFDFT_pc are compared with those deduced from the spectra. It is remarkable that the BLDFT values $\Delta_{1}$ and $\Delta_{2}$ compare nicely with experiment for most of the Ln complexes (they are underlined in Table 3, see also Figure 7). Exceptions are complexes of $\mathrm{Ce}, \mathrm{Pr}$, and $\mathrm{Nd}$ for which the LFDFT model still yields acceptable results. For these complexes ligand-to-metal charge transfer plays an important role. It follows that, within DFT, charge transfer is essential for the earlier members of the $\mathrm{Ln}$ series $(\mathrm{Ce}, \mathrm{Pr}, \mathrm{Nd})$, while it can be almost completely neglected for the rest of the 


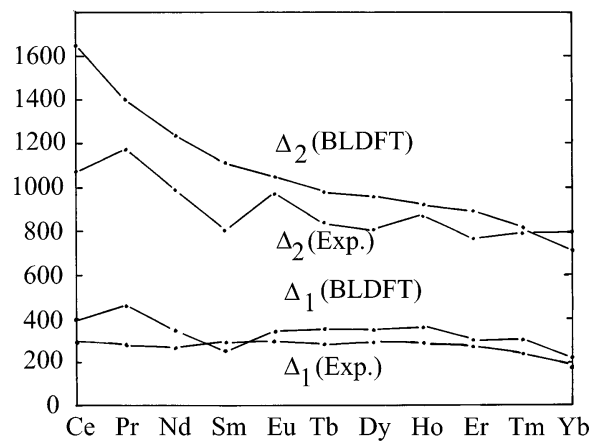

Figure 7. Values of $\Delta_{1}$ and $\Delta_{2}$ (in $\mathrm{cm}^{-1}$ ) from BLDFT calculations and from the interpretation of the $\mathrm{f}-\mathrm{f}$ spectra (experiment, ref 38 ) for $\mathrm{LnCl}_{6}{ }^{3-}$ complexes.

Table 6. Values of $\Delta_{1}$ and $\Delta_{2}$ (in $\mathrm{cm}^{-1}$ ), Resulting from Various DFT Computations for $\mathrm{YbBr}_{6}{ }^{3-}$ and 10Dq for $\mathrm{CrCl}_{6}{ }^{3-}$

\begin{tabular}{llrrrrr}
\hline complex & & LFDFT & BLDFT & CFDFT & CFDFT_pc & expt \\
\hline $\mathrm{YbBr}_{6}{ }^{3-a}$ & $\Delta_{1}$ & 2130 & 394 & 94 & 78 & $234^{c}$ \\
& $\Delta_{2}$ & 2333 & 908 & 148 & 158 & $641^{c}$ \\
$\mathrm{CrCl}_{6}{ }^{3-b}$ & $10 \mathrm{Dq}$ & 11345 & 2094 & -936 & 782 & $12800^{d}$
\end{tabular}

${ }^{a}$ Charge-compensated, $R(\mathrm{Yb}-\mathrm{Br})=2.83 \AA .{ }^{b}$ Charge-compensated; $R(\mathrm{Cr}-\mathrm{Cl})=2.335 \AA$. ${ }^{c}$ Reference $42 \mathrm{~b} .{ }^{d}$ Reference 43 .

series. Both $\Delta_{1}$ and $\Delta_{2}$ resulting from CFDFT calculations (Table 3) are comparably small, implying that Coulomb repulsion effects only are not the main source of the LF splitting of the Ln complexes. They are even calculated to yield a reverse ordering (small and negative $\Delta_{1}$ and $\Delta_{2}$ ) in the case of $\mathrm{Ce}, \mathrm{Pr}$, and $\mathrm{Nd}$. Approximating ligands in terms of negative point charges (CFDFT_pc, Table 3) leads to positive $\Delta_{1}$ and $\Delta_{2}$; i.e., to a correct orbital level ordering but numerical values are again distinctly lower than experimental ones. It is the Pauli (exchange) repulsion (BLDFT) which dominates in $\Delta_{1}$ and $\Delta_{2}$.

III.4. Discussion. The proposed approaches for calculating the one-electron LF matrix within KSDFT-LFDFT and BLDFT are easy to perform (using the ADF program) and can be applied to other systems. Different from the combined ligand field and density functional theory, ${ }^{20}$ in which electron density of the ligands is explicitly used to construct a pseudopotential for the $f$ electrons with parameters adjusted to experimental data, both the LFDFT and BLDFT methods are parameter-free. Our results for $\mathrm{YbBr}_{6}{ }^{3-}$ (BLDFT, Table 6) show a better correspondence between theoretical and experimental values of $\Delta_{1}$ and $\Delta_{2}$ for BLDFT, i.e., when excluding contributions due to ligand-to-metal charge transfer. This is similar to $\mathrm{YbCl}_{6}{ }^{3-}$ as well as to the other complexes of the latter Ln's. Turning to complexes of the $3 \mathrm{~d}$ series, the situation changes completely, however. In Table 6 10Dq values for $\mathrm{CrCl}_{6}^{3-}$ resulting from the various DFT-based computational schemes to obtain $\mathbf{V}_{\mathbf{L F}}$ are listed. Here comparison with experiment shows that the full LFDFT calculation of $10 \mathrm{Dq}$ yields better results. As in the case of the early $\mathrm{LnCl}_{6}{ }^{3-}$ complexes $(\mathrm{Ln}=\mathrm{Ce}, \mathrm{Pr}, \mathrm{Nd})$, ligand-tometal charge transfer is essential here and even dominates over both the Pauli(exchage repulsion) and the Coulombic effects, thus being the main factor which determines 10Dq. This is in line with earlier discussions of the origin of the
LF for $3 \mathrm{~d}, 4 \mathrm{~d}$, and $5 \mathrm{~d}$ TM complexes as due to metal-ligand covalency. ${ }^{31}$ Yet another manifestation of this observation is the nephelauxetic effect-the lowering of integrals of interelectronic repulsion in complexes compared to those of the free TM ions (see also the discussion in ref 21a).

The BLDFT scheme is particularly suited for cases in which, such as in latter Ln complexes, ionic metal-ligand interactions are dominating. The neglect of charge transfer between the metal and ligands turns out to be reasonable here and is equivalent to freezing out the electron density on the ligands, while treating their influence on the TM quantum mechanically. The BLDFT approach can be thus related to the orbital-free embedding potential derived as the consequence of the Hohenberg-Kohn variational principle which leads to Kohn-Sham-like one-electron equations for the orbitals representing the embedded subsystem. ${ }^{29 a}$

Finally, the good agreement between our BLDFT results for the latter Ln's allows us to state that pseudopotential theory ${ }^{44}$ when applied to the $\mathrm{LF}$ of $\mathrm{Ln}(\mathrm{Ln}=\mathrm{Sm}$ to $\mathrm{Yb})$ complexes, bears physical significance. These results show the potential of modeling the multiplet structure of $4 \mathrm{f}^{n}$ and $3 \mathrm{~d}^{n}$ configurations using atomic-like DFT programs to reduce significantly the computational efforts in more complex systems. Studies in that direction are in progress. ${ }^{45}$

\section{Conclusions and Outlook}

(1) Our analysis of $\mathrm{f}-\mathrm{f}$ and charge-transfer spectra of $4 \mathrm{f}$ $\mathrm{LnCl}_{6}{ }^{3-}$ have shown that mixing of $\mathrm{Cl} 3 \mathrm{p}$ into f orbitals does not exceed $1 \%$. Comparing this result with Kohn-Sham calculations for the same systems permits us to conclude that mixing between metal $4 \mathrm{f}$ and $\mathrm{Cl} 3 \mathrm{p}$ orbitals for nearly all the $\mathrm{Ln}$, except for $\mathrm{Ce}, \mathrm{Pr}$, and $\mathrm{Nd}$, are largely overestimated by Kohn-Sham orbitals, obtained using the currently available approximations to the exchange-correlation potential. They lead to artificially high values of LF splitting of the $4 \mathrm{f}$ orbitals. In this work, we propose a model (BLDFT) in which such contributions are excluded, combining thus the advantages of the Kohn-Sham formalism and the description of the ligands field by means of a pseudopotential.

(2) The comparison of the results obtained from KohnSham orbitals based ligand field theory (LFDFT) and the BLDFT scheme with experiment allows exploration of the role of ligand-to-metal charge transfer for energies of $\mathrm{f}-\mathrm{f}$ and $\mathrm{d}-\mathrm{d}$ transitions and to adjust KSDFT from some drawbacks connected with overdelocalization due to the exchange and correlation functionals in use. While for complexes of $\mathrm{Ce}, \mathrm{Pr}$ and $\mathrm{Nd}$ both ligand-to-metal charge transfer and exchange(Pauli) repulsion are shown by our calculations to play a significant role, for the latter members

(42) (a) Schwartz, R. W. Inorg. Chem. 1977, 16, 1694. (b) Tanner, P. A. Mol. Phys. 1986, 58, 317.

(43) Schwartz, R. W. Inorg. Chem. 1976, 15, 2817.

(44) (a) Vaidehi, N.; Wesolowski, T. A.; Warshel, A. J. Chem. Phys. 1992 97, 4264. (b)Watson, S. C.; Carter, E. A. Phys. Rev. B 1998, 58 R13309. (c) Zhou, B.; Wang, Y. A.; Carter, E. A. Phys. Rev. B 2004, 69, 125109.

(45) (a) Daul, C. XATOM - a DFT Computer Code for Atomic and Atomiclike Ligand Field Calculations. Université de Fribourg Suisse, Fribourg, Switzerland, 2004. Available from the author upon request. (b) GarciaLastra, J. M. Personal communication. 
of the $\mathrm{Ln}$ series $(\mathrm{Ln}=\mathrm{Sm}$ to $\mathrm{Yb})$, Pauli repulsion seems to play a decisive role. For all $\mathrm{LnCl}_{6}{ }^{3-}$ complexes crystal-fieldlike contributions to the LF have been calculated to be less important, contrary to usual assumptions implied when interpreting spectra of Ln in terms of CFT-like models. The first calculation to $\mathrm{CrCl}_{6}{ }^{3-}$ yields result in lines with the usual considerations: dominance of ligand field splitting by metalligand covalency (charge transfer).

(3) The results from our study lend support to recent parametrizations on the LF for rare earths, the simple overlap model, ${ }^{14,15}$ and the combined ligand field-density functional theory pseudopotential method, ${ }^{20}$ both combining features of the CF theory with a more adequate description which takes metal-ligand overlap into account.

(4) The simple BLDFT procedure proposed in this study is very useful for making analysis and comparing the results with the more rigorous, first-principles orbital-free embedding method. ${ }^{29}$
(5) We should finally note that the LFDFT model will intrinsically fail in cases (not encountered for the complexes considered in this study) where, because of low or even inverse (negative) ligand-to-metal charge transfer energy, the nature of one or more KS orbitals as being dominated by $4 \mathrm{f}$ functions will be highly violated. In cases such as this, and because of the decontamination procedure adopted, the BLDFT model can still be applied. However, situations of that type are very unlikely.

Acknowledgment. This work was supported by the Swiss National Science Foundation.

Supporting Information Available: Additional details and tables (PDF). This material is available free of charge via the Internet at http://pubs.acs.org.

IC040105T 